\title{
Protection from premature habituation requires functional mushroom bodies in Drosophila
}

\author{
Summer F. Acevedo, ${ }^{1,2}$ Emmanuil I. Froudarakis, ${ }^{1}$ Alexandros Kanellopoulos, ${ }^{1}$ \\ and Efthimios M.C. Skoulakis ${ }^{3}$ \\ Institute of Molecular Biology and Genetics, Biomedical Science Research Centre "Alexander Fleming," Vari 16672, Greece
}

\begin{abstract}
Diminished responses to stimuli defined as habituation can serve as a gating mechanism for repetitive environmental cues with little predictive value and importance. We demonstrate that wild-type animals diminish their responses to electric shock stimuli with properties characteristic of short- and long-term habituation. We used spatially restricted abrogation of neurotransmission to identify brain areas involved in this behavioral response. We find that the mushroom bodies and, in particular, the $\boldsymbol{\alpha} / \boldsymbol{\beta}$ lobes appear to guard against habituating prematurely to repetitive electric shock stimuli. In addition to protection from premature habituation, the mushroom bodies are essential for spontaneous recovery and dishabituation. These results reveal a novel modulatory role of the mushroom bodies on responses to repetitive stimuli in agreement with and complementary to their established roles in olfactory learning and memory.
\end{abstract}

Organisms must be able to assign importance to novel and preexperienced stimuli and evaluate their significance as a prerequisite to selection of the appropriate response. Habituation is a major mechanism to decrease responsiveness to repetitive or prolonged nonreinforced stimuli (Thomson and Spencer 1966; Groves and Thomson 1970; Rankin 2000; Rose and Rankin 2001). Because response attenuation depends on prior experience of a stimulus, habituation has been studied as a form of nonassociative learning in a variety of models (Hawkins 1988; Burrell and Sahley 1998; Rose and Rankin 2001; Deshmukh and Bhalla 2003; Ezzeddine and Glanzman 2003). Furthermore, since habituation may underlie selective attention (Groves and Thomson 1970; Freedman et al. 1991; Gillberg 2003), its premature onset could impair adequate discrimination between novel and preexperienced stimuli. Habituation deficits have been associated with schizophrenia (Freedman et al. 1991; Adler et al. 1999; Gillberg 2003; Meincke et al. 2004), learning disabilities (Gillberg 2003; Slaats-Willemse et al. 2003), and migraines (Siniatchkin et al. 2003), among other conditions.

In Drosophila, aspects of habituation have been investigated using several diverse assays (Duerr and Quinn 1982; Corfas and Dudai 1989; Asztalos et al. 1993; Engel and Wu 1996; Jin et al. 1998; Engel et al. 2000; Cho et al. 2004). With the exception of the proboscis extension reflex (Duerr and Quinn 1982), most assays focused on habituation of peripheral nervous system (PNS) sensory neurons. Little has been done to investigate habituation mediated by the central nervous system (CNS) (Cho et al. 2004) and, in particular, stimuli used for the prevalent paradigms of associative conditioning in this insect. To investigate CNSmediated habituation and the anatomical sites that mediate it, we focused on whether Drosophila could habituate to electric shocks, the stimulus typically used as the unconditioned stimulus in the prevalent negatively reinforced associative learning and memory assay (Tully and Quinn 1985). Choice of this stimulus was advantageous because shock avoidance is a robust re-

\footnotetext{
1These authors contributed equally to this work.

2Present address: Oregon Health Sciences University, Behavioral Neuroscience L-470, 3181 SW Sam Jackson Park Road, Portland, OR 97239, USA.

${ }^{3}$ Corresponding author.

E-mail skoulakis@fleming.gr; fax 30-210-965-6563.

Article is online at http://www.learnmem.org/cgi/doi/10.1101//m.566007.
}

sponse (Tully and Quinn 1985; Skoulakis et al. 1993; Skoulakis and Davis 1996), the number and strength of stimuli could be easily and reliably quantified and adjusted, and an experimental apparatus was readily available since the assay could be conducted in a standard T-maze. Significantly, since electric shock is used as a stimulus for associative conditioning that depends on the mushroom bodies (Heisenberg 2003; McGuire et al. 2005; Skoulakis and Grammenoudi 2006), it was hypothesized that electric shock stimuli likely converge within these neurons with the odor stimuli for learning to occur. Therefore, the mushroom bodies (MBs) were an obvious neuroanatomical site potentially involved in the behavioral response under consideration. Moreover, habituation to shock could provide comparisons of associative and nonassociative processes and insights into the deficits of extant and novel learning mutants with at least one identical stimulus.

The MBs are bilateral neuronal clusters comprised of $\sim 2500$ neurons each, in the dorsal posterior cortex of the brain (Roman and Davis 2001; Heisenberg 2003). The dendrites (calyces) lie ventrally to the somata of these neurons, while their axons fasciculate, forming the anteriorly projecting pedunculus, where it bifurcates to medially extending ( $\beta, \beta^{\prime}$, and $\gamma$ lobes) and dorsally projecting processes ( $\alpha$ and $\alpha^{\prime}$ lobes) (Crittenden et al. 1998; Heisenberg 2003). Olfactory information reaches the MBs via at least one major ascending tract of projection neurons connecting the antennal lobes with the MBs and Lateral Horn, the inner Antennal-Cerebral Tract (Stocker et al. 1997). Electric shock to the abdomen has been shown to activate multiple synapses in the Drosophila brain (Yu et al. 2004), but it is not currently known whether a dedicated ascending neuronal tract delivers information to the MBs. The MBs were reported to participate in habituation to an aversive odor (Cho et al. 2004), but this could be related to their role in olfactory information processing (Tanaka et al. 2004) and may not be a general property of these neurons. We address this question by investigating whether the MBs are important for habituation to electric shock and demonstrate that these neurons are essential in preventing premature habituation.

\section{Results}

Habituation to repetitive electric shocks

To determine whether Drosophila could habituate to electric shocks, we exposed $w^{1118}$ and Berlin $(B)$ animals, two control 
strains with different genetic backgrounds, to repeated $45 \mathrm{~V}$ electrical pulses. We adopted the $45 \mathrm{~V}$ stimuli for training and testing because at this strength, shocks elicit relatively mild, yet consistent responses (Fig. 1C,D). The training phase of the experiment consisted of trapping animals on an electrifiable grid and exposing them to one to 15 electric shocks delivered within a 60-sec training period. Approximately two minutes after training, the flies were given a choice between an inert grid and an identical one, electrified for the 90 -sec testing period with stimuli of the same frequency and duration as for training. The number of shocks and interval between them were determined experimentally as the conditions that generated maximal avoidance of the electrified grid in naive animals.

Naive animals and ones exposed to one to four shocks during training avoided the electrified grid robustly during testing. Surprisingly, experience of eight or more stimuli attenuated avoidance of the electrified grid during testing, with maximal attenuation reached after exposure to 11 or more shocks (Fig. 1A). Therefore, prior experience of 11-15 unpaired shocks resulted in more than twice as many flies staying on the electrified grids at the end of the test period compared to naive individuals. The distribution of animals on the electrified and inert grids after training did not change significantly if more or less time was allocated for testing (data not shown). This indicated that the experience-dependent attenuation of shock avoidance was not a consequence of impaired locomotion elicited by training. If locomotion was affected by training, a significant fraction of animals would be expected to remain in the central compartment of the maze, which was not observed.

Shock avoidance recovered spontaneously to naive levels if the flies were allowed at least 6 min of rest after training (Fig. 1B), as predicted if the response decrement was due to habituation (Thomson and Spencer 1966; Groves and Thomson 1970; Rankin and Broster 1992). Furthermore, if animals were trained and tested with stronger stimuli $(60 \mathrm{~V}$ and $90 \mathrm{~V})$, the magnitude of shock avoidance attenuation was significantly reduced (Fig. $1 \mathrm{C}, \mathrm{D})$. Therefore, the experience-dependent reduction in shock avoidance was inversely correlated to the strength of the stimulus. These results suggest that the experience-dependent attenuation of shock avoidance could be the outcome of habituation to the multiple electric shocks during training, fatigue, or desensitization.

If the experience-dependent decline of shock avoidance was habituation and not sensory desensitization or fatigue, the effect should be eliminated by brief application of an unrelated noxious stimulus (dishabituation) (Thomson and Spencer 1966; Groves and Thomson 1970). Therefore, we attempted to eliminate the effects of shock pre-exposure with a strong olfactory stimulus delivered at the end of the training episode. Animals exposed to the aversive odor benzaldehyde (BNZ) for $10 \mathrm{sec}$ immediately after training avoided test shocks indistinguishably from naive animals. BNZ exposure alone did not affect subsequent shock avoidance (Fig. 1E). These results support the argument that the experience-dependent attenuation of shock avoidance is not the result of desensitization or fatigue, but rather habituation to shocks during training (Groves and Thomson 1970; Marcus et al. 1988; Rankin and Broster 1992; Rose and Rankin 2001), and exposure to BNZ dishabituates the response. Similar results were obtained if a different aversive odor, 3-octanol, was used as the dishabituator (data not shown). Interestingly, although it is also a moderate aversive stimulus that has been successfully used to dishabituate Drosophila (Mihalek et al. 1997; Cho et al. 2004), mechanical stimulation (shaking) failed to dishabituate the response (Fig. 1E). This suggests that perhaps electric shocks may be perceived as a mechanical stimulation and because habituation to electric shock may generalize to other
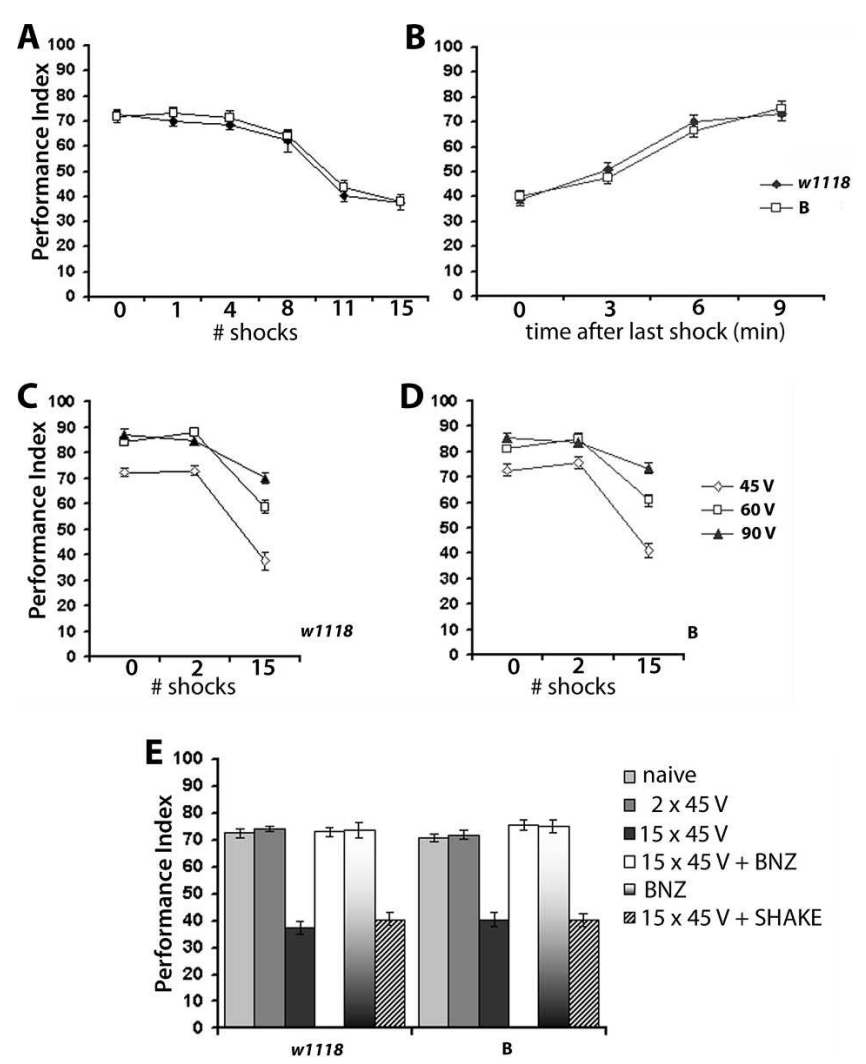

Figure 1. Habituation to repeated exposure to electric shock. (A) Experience-dependent attenuation of shock reactivity. Avoidance of a copper grid where $45 \mathrm{~V}$ shocks are delivered every $4 \mathrm{sec}$ with $1.2 \mathrm{sec}$ duration each, for the 90 -sec test period. 0 denotes avoidance of the electrified grid without prior experience of similar electric shocks (naive), whereas the numbers indicate the number of shocks experienced prior to testing. Each measurement represents the mean performance indices $(\mathrm{PI}) \pm$ standard error of the mean (SEM) for $n \geq 8$ for each wild-type stock; (filled diamonds) $w^{1118}$; (open squares) Berlin (B). ANOVA indicated significant differences $\left(F_{(11,127)}=39.46 ; P<0.0001\right)$ among the Pls. Subsequent planned comparisons indicated significant differences $(P<0.001)$ in shock avoidance of naive animals and the performances after pre-exposure to 11 and 15 shocks. Performance after eight shocks was also different from that of naive animals $(P<0.05)$. (B) Spontaneous recovery of shock avoidance following exposure to 15 training shocks. $\mathrm{PI} \pm$ SEMs are shown for $n \geq 8$, per time interval for each genotype. ANOVA $\left(F_{(7,84)}=12.37 ; P<0.001\right)$ indicated significant differences. Planned comparisons revealed highly significant differences $(P<0.001)$ in shock avoidance after 6 and 9 min of rest in comparison to that immediately after training with 15 shocks (0). These animals are distinct from those used in part $A$, but because the experiments in $A$ and $B$ were performed concurrently, we also compared the performance of naive animals $(0$ in $A)$ to that that of animals trained with 15 stimuli and allowed to rest for $9 \mathrm{~min}$. These two means were not found to be statistically different $(P<0.1336)$. $(C, D)$ Animals were trained and tested to the same stimulus strength. The magnitude of shock avoidance decrement is inversely related to stimulus strength. $\mathrm{PI} \pm$ SEMs are shown for $n \geq 8$ per group. Naive avoidance of $45 \mathrm{~V}$ shocks was different (planned comparisons, $P<0.001)$ from those at 60 and $90 \mathrm{~V}$ for both $w^{1118}(C)$ and $B(D)$. Avoidance of $45 \mathrm{~V}$ shocks after exposure to 15 stimuli at $45 \mathrm{~V}$ was significantly different from that at 60 or $90 \mathrm{~V}$ (planned comparisons, $P<0.001)$ for $w^{1118}(C)$ and $B(D)$. Similarly, avoidance of $60 \mathrm{~V}$ shocks after exposure to 15 stimuli at $60 \mathrm{~V}$ was significantly different from that at $90 \mathrm{~V}$ (planned comparisons, $P<0.001)$. ( $E$ ) Reversal of the experiencedependent attenuation of shock avoidance with an olfactory stimulus. $\mathrm{PI} \pm$ SEMs are shown for $n \geq 8$ per group. ANOVA $\left(w^{1118}\right)$, $F_{(5,57)}=31.26 ; P<0.0001 ; \operatorname{ANOVA}(B), F_{(5,55)}=24.17 ; P<0.0001$. Subsequent Dunnett's tests revealed that compared to the performance of naive animals, exposure to 15 training stimuli significantly $(P<0.001)$ altered shock avoidance during testing, but two training stimuli did not. Significantly, shock avoidance of animals trained with 15 stimuli, but exposed to $10 \mathrm{sec}$ of benzaldehyde prior to testing $(15 \times 45 \mathrm{~V}+\mathrm{BNZ})$, performed indistinguishably from control animals. The performance of animals shaken after training with 15 stimuli was significantly different $(P<0.001)$ from that of naive animals. 
mechanosensory stimuli, shaking is not an appropriate dishabituator (Groves and Thomson 1970; Mackintosh 1974). Furthermore, a single $90 \mathrm{~V}$ electric shock at the end of training with 45 $\mathrm{V}$ stimuli did not ameliorate the response attenuation (data not shown), suggesting generalization of the response to shocks of different strengths. As expected, if the reduction in shock avoidance represented a habituated response, the decrement was apparent after experiencing a distinct number (eight to nine) of training stimuli, indicating a minimal requirement for repetitive stimulation before the habituation becomes apparent. Similar refractory periods have been observed in other habituation protocols for Drosophila (Corfas and Dudai 1989; Boynton and Tully 1992; Engel and $\mathrm{Wu}$ 1996) and other invertebrates (Hawkins 1988; Rankin and Broster 1992; Beck and Rankin 1997; Rose and Rankin 2001) and in agreement with classically defined habituation characteristics (Thomson and Spencer 1966).

\section{Long-term habituation}

Repeated training cycles have been reported to precipitate a longlasting habituated response (Beck and Rankin 1997; Rose and Rankin 2001). Therefore, we investigated whether multiple cycles of training with 15 stimuli resulted in long-lasting attenuation of shock avoidance. In comparison to the response of naive animals, shock avoidance remained significantly different $30 \mathrm{~min}$ after even a single training cycle (Fig. 2A). Additional training cycles delivered with an ITI of 5 min did not have a significant effect on performance tested immediately after the last cycle. However, three training cycles yielded significantly more attenuation of shock avoidance $30 \mathrm{~min}$ later. To ascertain that the increased attenuation of shock avoidance was not a consequence of fatigue, we delivered a 10-sec BNZ pulse after the third training cycle. Shock avoidance tested immediately following the odor pulse or $30 \mathrm{~min}$ later was not significantly different from that exhibited by naive flies (Fig. 2A), indicating that the multiple training cycles did not fatigue the animals, but, rather, established deeper and longer lasting habituation. Memory of the attenuated shock avoidance could be detected $60 \mathrm{~min}$ after four training cycles with a 5-min ITI, or for $90 \mathrm{~min}$ after four cycles with a 10-min ITI (Fig. 2B). In fact, memory was better at all tested retention intervals after four cycles of training with 10min ITI, consistent with the habituation parameters described in previous studies (Beck and Rankin 1997; Rose and Rankin 2001).

Collectively, the results indicate that the experiencedependent attenuation of shock avoidance conforms fully to habituation parameters and define a novel paradigm of nonassociative learning and memory in Drosophila.

\section{Abrogation of mushroom body function precipitates rapid attenuation of shock avoidance}

We attempted to identify the locus in the brain necessary for habituation to electric shock by inhibiting neurotransmission in specific groups of neurons with tetanus toxin light chain (TNT) expression with spatially restricted GAL4 drivers (Sweeney et al. 1995; Keller et al. 2002). Because the mushroom bodies (MBs) are central to information processing underlying associative learning and memory, and electric shocks are thought to engage the MBs (Davis 2005; Skoulakis and Grammenoudi 2006), we used the MB-specific driver P247 (Zars et al. 2000) to inhibit neurotransmission from these neurons. We also used the GH146 driver to inhibit neurotransmission from the iACT connecting the antennal lobes with the MBs (Stocker et al. 1997). iACT synapses were shown to be activated by electric shock to the abdomen (Yu et al. 2004). In addition, we used driver c507, which specifically marks neurons of the ellipsoid body (Yang et al. 2000), a brain area that is likely involved in regulating aspects of locomotion (Ilius et al.
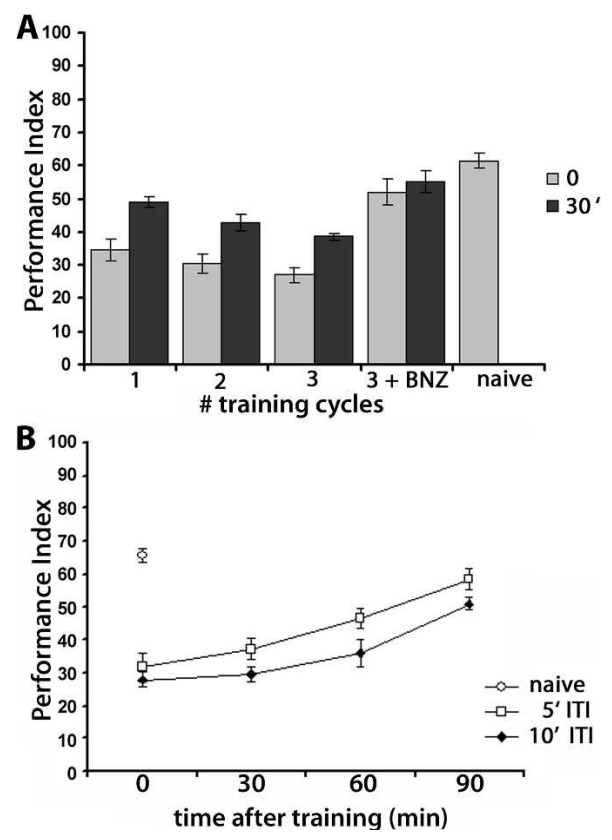

Figure 2. Repeated training cycles lead to long-term habituation. $(A)$ The effect of repeated training cycles on habituation measured immediately after training and 30 min post-training. PI \pm SEMs are shown for $n \geq 8$ per group. ANOVA indicated significant differences: $F_{(8,61)}=21.09$, $P<0.0001$. Subsequent Dunnett's tests revealed highly significant differences $(P<0.0001)$ in the performance of naive animals compared to that of animals trained with one, two, or three cycles immediately after training or 30 min later. In contrast, the performance of animals tested after three cycles of training followed by a single exposure to benzaldehyde was not significantly different immediately $(P=0.214)$ or 30 min later $(P=0.101)$ from that of naive animals. $(B)$ Robust habituation is detected after four training cycles with a 10-min ITI for at least 90 min. PI \pm SEMs are shown for $n \geq 7$ per group. ANOVA indicated significant effects of ITI and retention interval $(P<0.001)$. Subsequent planned comparisons revealed that for ITIs of $5 \mathrm{~min}$, performance at all retention intervals except 90 min post-training were significantly different from the performance of naive animals. Similarly, for ITI of $10 \mathrm{~min}$, performances at all retention intervals were significantly different $(P<0.001)$ from that of naive animals.

1994; Martin et al. 1999, 2001) and may be required for manifestation of the attenuated response. Finally, we used driver 78Y, which is expressed in particular structures of the central complex, the protocerebral bridge, ellipsoid body, and noduli (Martin et al. 1999), neurons essential for locomotion and coordination in the fly (Strauss et al. 1992; Martin et al. 1999).

We used heterozygotes for all GAL4 driver insertions as controls and to ascertain that each transposon insertion did not precipitate defects on its own when heterozygous. Animals expressing TNT in the brain areas described above and controls exhibited similar shock avoidance reduction following 15-stimulus training, suggesting that inhibition of neurotransmission in these neurons did not inhibit or delay habituation. However, significantly attenuated shock avoidance was detected in animals with silenced $\mathrm{MB}$ synapses even after training with only two stimuli (Fig. 3). This phenotype was not observed in any of the control animals, or animals with blocked neurotransmission in the iACT, ellipsoid body, protocerebral bridge, or noduli. Therefore, blocking neurotransmission in the MBs resulted in faster habituation, suggesting a significant reduction in the refractory period. To verify this independently, we used the mutant $\mathrm{mbm}^{1}$, which harbors small and deranged MBs (Heisenberg et al. 1985; Acevedo et al. 2007). Both $\mathrm{mbm}^{1}$ homozygotes and P247 > UASTNT animals avoided the electric shocks normally when naive $(0$ 
shocks in Fig. 4A). However, even a single shock prior to testing attenuated shock avoidance significantly in comparison to their respective control strains. The decline in shock avoidance in $\mathrm{mbm}^{1}$ and P247>UAS-TNT animals reached a stable plateau after a second training stimulus (Fig. 4A). In contrast, control animals exhibited habituated responses after 10 training stimuli, and their performance equaled that of the MB-perturbed animals after training with 15 stimuli (Fig. 4A). Therefore, structural or functional perturbation of the MBs appears to result in premature habituation to electric shocks.

\section{The mushroom bodies are required for spontaneous recovery and dishabituation}

To determine whether the rapid attenuation of shock avoidance represented a habituated response, we investigated whether animals with perturbed MBs recover spontaneously. We elected to use two stimuli during training, because they induce as strong an attenuation of shock avoidance in P247>UAS-TNT and $\mathrm{mbm}^{1}$ animals, as 15 stimuli do in controls (Fig. 4A). In fact, experience of two training stimuli precipitated the attenuated response, whereas the performance of controls remained unchanged (Fig. 4B). The shock avoidance attenuation exhibited by MB-perturbed animals was detectable 6 min after training, but had decayed to control levels after 15 min (Fig. 4B). In contrast, complete spontaneous recovery of shock avoidance in control strains was observed within 6 min after training with 15 stimuli (Fig. 1B). Therefore, attenuation of shock avoidance is established more rapidly in animals with perturbed MBs and recovers spontaneously, albeit more slowly compared to controls. It appears then that functional MBs are required for normal spontaneous recovery.

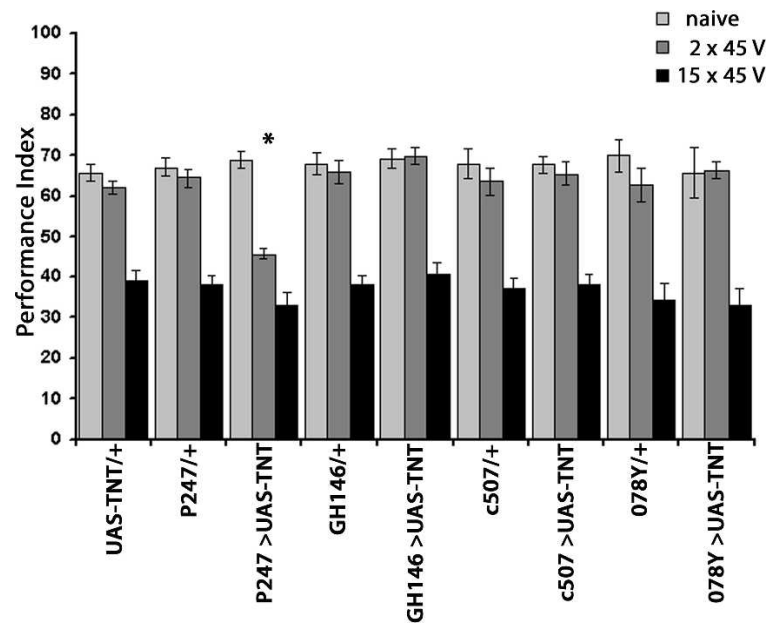

Figure 3. Impairing neurotransmission in different parts of the brain reveals that the $\mathrm{MBs}$ are involved in protection from premature habituation to electric shock. PI \pm SEMs are shown for $n \geq 7$ per group. The UAS-TNT/+ group was included in this experiment simply to demonstrate that this transgene alone did not precipitate significant behavioral alterations. However, since all strains expressing TNT under the drivers used (signified by $>$ ) were accompanied by control strains carrying the driver transgene as a heterozygote in a balanced experimental design. Significant differences in the performance of all strains when naive were not observed (ANOVA: $F_{(8,89)}=2.37 ; P<0.0123$ ). However, significant differences in subsequent shock avoidance were revealed for animals trained with two stimuli (ANOVA: $F_{(8,82)}=12.41 ; P<0.0001$ ). This difference arose from the highly significant difference in avoidance of P247 > UASTNT animals compared to their P247/+ controls $(P<0.001$, Student's $t$-test). No significant differences in the performance of all strains were detected after training with 15 stimuli (ANOVA: $F_{(9,87)}=1.28$; $P<0.0598$ ).
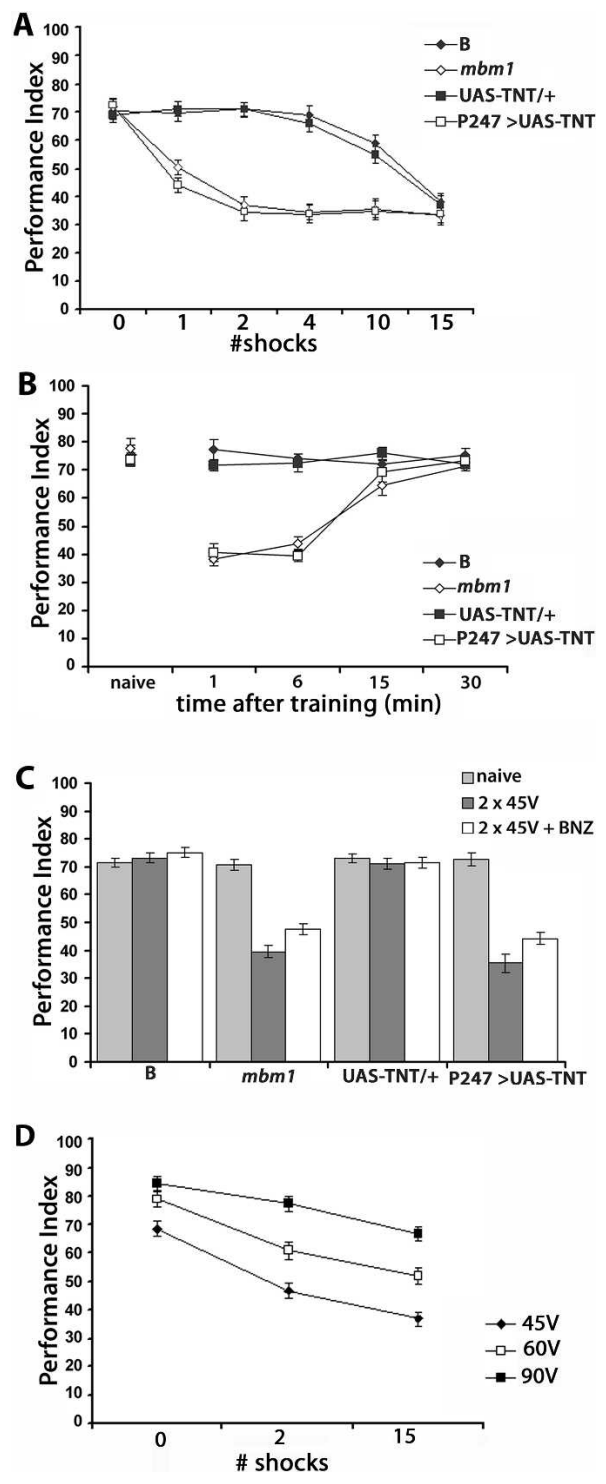

Figure 4. (Legend on next page)

The rapid decrease in shock avoidance was not reverted to naive levels with exposure to BNZ post-training, although both $\mathrm{mbm}^{1}$ and P247>UAS-TNT flies exhibited a trend of higher shock avoidance after BNZ exposure (Fig. 4C). This was not a consequence of MB-perturbed animals being unable to sense and respond normally to the odor (deBelle and Heisenberg 1994). Shaking or exposure to bright light also did not result in normal post-training shock avoidance. Therefore, it appears that intact MBs are also required for normal dishabituation. Alternatively, because it could not be dishabituated, attenuation of shock avoidance in animals with structurally or functionally perturbed MBs may not reflect bona fide habituation, but, rather, fatigue or desensitization. We think the latter is not the case for two reasons. First, it is unlikely that two training stimuli suffice to precipitate fatigue or desensitization in animals with perturbed MBs, when 15 or more such stimuli do not in controls. Moreover, if the attenuated response was fatigue or desensitization, it would be expected to be exaggerated by stronger stimulation. However, exposure of $\mathrm{mbm}^{1}$ animals to one or 15 shocks of 60 or $90 \mathrm{~V}$ did not result in deeper or faster attenuation of the response. In fact, after 15 training stimuli, the largest response attenuation was 
Figure 4. Premature habituation to shock in animals with structurally or functionally perturbed MBs. $(A)$ Two training stimuli suffice to precipitate significant attenuation in shock avoidance in MB-perturbed animals. $\mathrm{PI} \pm$ SEMs are shown for $n \geq 7$. 0 represents shock avoidance of naive animals. ANOVA indicated significant effects of shock number and genotype $\left(F_{(23,176)}=38.61 ; P<0.0001\right)$. Compared to the performance of naive controls ( 0 time), significant differences $(P<0.001$, Dunnett's test) in performance arose after one training shock and remained highly significant in MB-perturbed animals. In contrast, 10 training stimuli were required for control strains to exhibit significantly $(P<0.001)$ attenuated performance compared to naive animals. $(B)$ Recovery of normal (naive) shock avoidance $15 \mathrm{~min}$ after training $\mathrm{MB}$-perturbed animals with two stimuli. PI \pm SEMs are shown for $n \geq 7$. The performance of naive animals of all relevant strains is shown as reference. ANOVA indicated significant effects of the rest interval and genotype $\left(F_{(19,154)}=46.61\right.$; $P<0.0001)$. Subsequent Dunnett's tests with the performance of naive animals of each strain used as control, revealed that following twostimulus training, the performance of MB-perturbed animals was significantly $(P<0.0001)$ attenuated after $6 \mathrm{~min}$, but not after $15 \mathrm{~min}$ of rest. In contrast, significant differences in shock avoidance of control strains were not detectable immediately, or at later rest intervals. (C) Naive shock avoidance was not restored by an odor pulse following two-stimulus training. $\mathrm{PI} \pm$ SEMs are shown for $n \geq 8$. Compared to the performance of naive flies, there was no significant difference in shock avoidance of $B$ and UAS-TNT/+ after two-stimulus training, or a BNZ pulse after training. In contrast, the performance of naive $\mathrm{mbm}^{1}$ and P247>UAS-TNT animals remained significantly different from that after two-stimulus training and after the BNZ pulse $(P<0.001)$. (D) The magnitude of shock avoidance attenuation is inversely related to stimulus strength. $\mathrm{PI} \pm \mathrm{SEM}$ s are shown for $\mathrm{mbm}^{7}$ animals, $n \geq 7$. ANOVA indicated significant effects of both stimulus strength and number of stimuli $\left(F_{(8,69)}=50.25\right.$; $P<0.0001)$. Subsequently, the performance of animals under $45 \mathrm{~V}$ stimulation was compared to those under 60 and $90 \mathrm{~V}$ (Hsu's test). Naive avoidance at 60 and $90 \mathrm{~V}$ was significantly different from that at $45 \mathrm{~V}$ $(P<0.001)$. Similarly, shock avoidances at 60 and $90 \mathrm{~V}$ were significantly different from those at $45 \mathrm{~V}$ after two- or 15-stimulus training $(P<0.001$ for both comparisons).

observed when animals were exposed to the milder $45 \mathrm{~V}$ stimuli (Fig. 4D). Therefore, the magnitude of shock avoidance decrease was inversely correlated to the strength of the training stimulus for animals with perturbed MBs as was described for controls (Fig. $1 C, D)$. This is consistent with the notion that the attenuated shock avoidance is the result of premature habituation and not fatigue or desensitization in MB-disturbed animals.

Collectively these data indicate that structural or functional perturbation of the MBs results in premature habituation to electric shock stimuli, and that these neurons are also important for normal spontaneous recovery and required for dishabituation.

\section{Protection from premature habituation to electric shock requires functional $\boldsymbol{\alpha} / \boldsymbol{\beta} \mathrm{MB}$ lobes}

We used additional drivers to express TNT in the MBs, as well as hydroxurea (HU) mediated ablation of these neurons (Acevedo et al. 2007) for two reasons: first, to independently verify their role in protection from premature habituation to shock; and second, to investigate whether this function could be localized to particular groups of cells within the MBs. We used the two-shock training protocol to induce attenuated shock avoidance.

All strains of MB-perturbed animals examined displayed normal habituation after training with 15 shocks (data not shown). In addition, accumulation of TNT in the MBs did not result in detectable morphological alterations of these neurons (data not shown; but see Thum et al. 2006). Similar to the phenotype of $\mathrm{mbm}^{1}$, ablation of the MBs resulted in robust attenuation of shock avoidance after training with two stimuli (Fig. 5A), verifying independently that these neurons are essential for protection from premature habituation. Shock avoidance in control and experimental animals was not significantly different when naive, indicating that intact MBs are not essential for shock avoidance per se. In addition to P247, we used driver c772, which is robustly expressed in $\alpha / \beta$ and to a lesser degree in $\gamma$ lobes (Mershin et al. 2004). TNT accumulation in these neurons also precipitated premature habituation after training with two shocks. To determine whether inhibition of neurotransmission in the $\alpha / \beta$ or the $\gamma$ lobe was responsible for the effect, we used driver H24. TNT expression under this driver in the $\gamma$ lobe, antennal lobes, and ellipsoid body (Zars et al. 2000) did not result in premature habituation (Fig. 5A). This result confirms data from Figure 3 regarding the ellipsoid body and indicates that the antennal lobe is not essential for the response. We also used the $201 Y$ driver, which is expressed in all types of MB neurons, but especially prominently in the $\gamma$ lobes (Yang et al. 1995). Interestingly, TNT expression under $201 \mathrm{Y}$ resulted in reduced shock avoidance in naive flies, probably a consequence of toxin accumulation in neurons outside the MBs (Michel et al. 2004). Nevertheless, shock avoidance was further attenuated in $201 \mathrm{Y}>\mathrm{UAS}$ TNT animals after training with two stimuli (Fig. 5A), suggesting in combination with the result with the $\mathrm{H} 24$ driver that premature habituation is mediated by neurotransmission blockade in the $\alpha / \beta$ lobes. Collectively these results suggest that neurotransmission from the $\mathrm{MB} \alpha / \beta$ lobes, but not the $\gamma$ lobe, is important for protection from premature habituation to electric shock.

To verify this conclusion, we sought to inhibit neurotransmission specifically in the $\alpha / \beta$ and $\gamma$ lobes. For the $\alpha / \beta$ lobes, we selected driver $11 \mathrm{Y}$ because of its specificity and robust expression in these neurons (http://www.fly-trap.org). Because TNT expression under $11 \mathrm{Y}$ precipitated lethality, we instead used the thermo-sensitive form of dynamin $\left(\mathrm{SHI}^{\mathrm{ts}}\right)$, which under nonpermissive conditions blocks neurotransmitter re-uptake, thus conditionally inhibiting neurotransmission (Kitamoto 2001). In addition to H24, we used the $\gamma$-lobe preferential driver 1471 (Isabel et al. 2004) and the mostly $\gamma$-lobe-specific (very low expression in $\alpha^{\prime} / \beta^{\prime}$ lobes) NP1131 (Akalal et al. 2006). Furthermore, neurotransmission was inhibited in P247 and in GH146 marked neurons as a positive and negative control, respectively, based on the results detailed above. We also included the $201 Y$ driver to verify independently the results obtained with TNT.

Silencing iACT synapses with GH146-driven UAS-shits ${ }^{\text {ts }}$ did not precipitate premature habituation to shock, in congruence with the results obtained by expressing TNT in these neurons (Fig. 3). In contrast, abrogation of neurotransmission in neurons marked by P247 resulted in premature habituation (Fig. 5B) as expected (Figs. 3, 5A). Once again, shock avoidance of $201 Y>U A S-s h i^{\text {ts }}$ was significantly reduced compared with their heterozygous controls, but as described above, was further reduced after two training shocks. Therefore, premature habituation to shock was reproduced by independent means of silencing particular groups of synapses. Significantly, inhibiting neurotransmission specifically via the $\alpha / \beta$ lobes with driver $11 \mathrm{Y}$ yielded strong premature habituation (Fig. 5B). In contrast, shi ${ }^{\text {ts }}$ mediated silencing of $\gamma$-lobe synapses with the H24, 1471, or the specific NP1131 driver did not precipitate premature habituation (Fig. 5B), although these strains exhibited normal habituation after 15 training stimuli (data not shown). These results confirm that $\gamma$ lobes are not essential and that neurotransmission from the $\alpha / \beta$ lobes to yet unknown follower neurons, is requisite for protection from premature habituation, normal spontaneous recovery, and dishabituation. Collectively, the data support the notion that the MBs are essential neuronal centers involved in evaluation and response to repetitive electric shock stimulation.

\section{Discussion}

Studies over the last 20 years have established that Drosophila habituate to stimuli in diverse experimental protocols such as the landing response (Rees and Spatz 1989; Asztalos et al. 1993), proboscis extension reflex (Duerr and Quinn 1982), cleaning reflex 

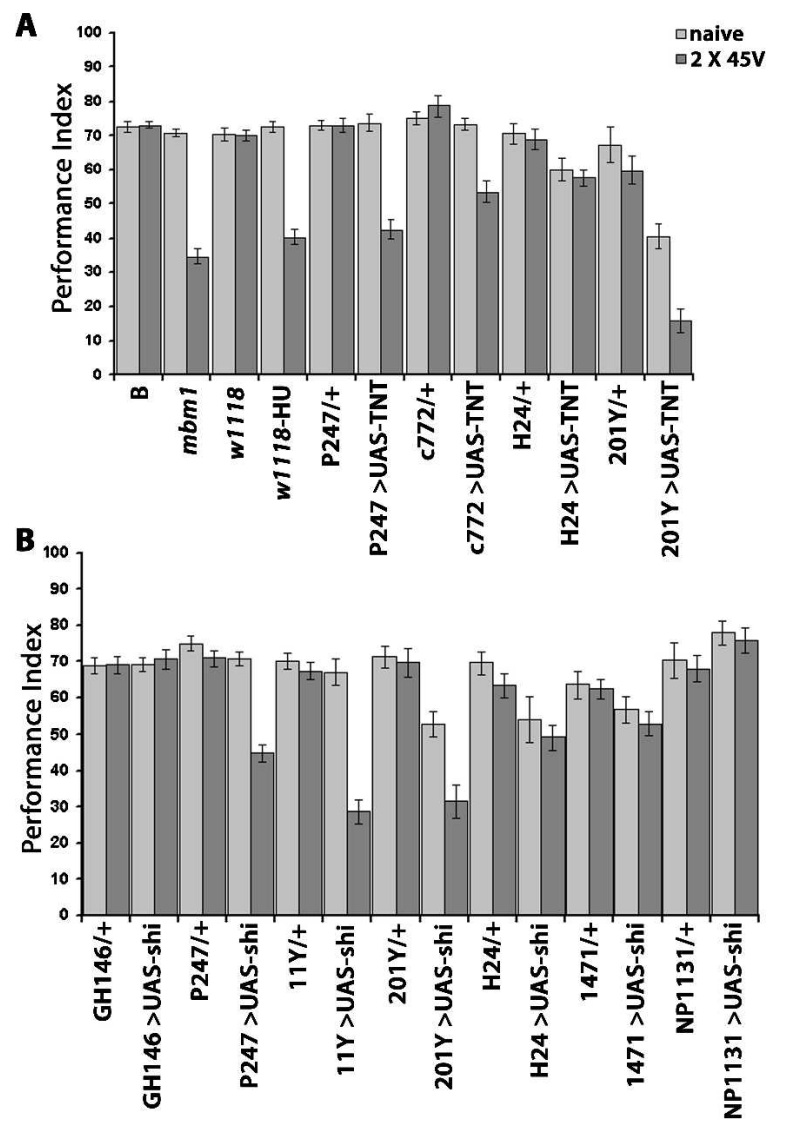

Figure 5. Protection from premature habituation to shock requires the $\alpha / \beta$ lobes of the MBs. (A) Structural and functional ablation of the MBs precipitates habituation to shock after two training stimuli. PI \pm SEMs are shown for $n \geq 7$. The performance of all experimental strains was compared to their relevant heterozygous controls. There were no significant differences in naive avoidances except between 201Y/+ and the experimental $201 Y>$ UAS-TNT $(P<0.001)$. Two-stimulus training yielded significant differences in the performance of $\mathrm{mbm}^{1}$, compared to that of $B$; $w^{1118}-H U$, compared to $w^{1118}$ alone; P247>UAS-TNT compared to P247/ +; 201Y>UAS-TNT, compared to 201Y/+; and c772>UAS-TNT, compared to $c 772 /+(P<0.001$ for all comparisons, Student's $t$-tests $)$. (B) Inhibition of neurotransmission specifically in the $\alpha / \beta$ lobes leads to premature habituation to shock. PI \pm SEMs are shown for $n \geq 7$. The performance of all strains after incubation at $32^{\circ} \mathrm{C}$ (see Materials and Methods) is shown. The performances of animals expressing UAS-shits under P247, $11 Y$, and $201 Y$ after training with two stimuli were significantly different from those of their respective control heterozygotes $(P<0.001$, Student's $t$-tests). In contrast, the performances of animals expressing UAS-shits under $\mathrm{GH} 146, \mathrm{H} 24,1471$, and NP1131 were not significantly different from those of their respective controls.

(Corfas and Dudai 1989), olfactory startle (Mihalek et al. 1997; Cho et al. 2004), visual startle reflex (Engel and Wu 1996; Engel et al. 2000), and leg position (Jin et al. 1998). In general, these paradigms used single animals, often restrained or decapitated, and many focused on proprioceptive reflexes of thoracic sensory neurons, coupling behavioral manipulation and electrophysiology (Corfas and Dudai 1989; Engel and Wu 1996; Jin et al. 1998; Engel et al. 2000). We have established a simple, populationbased paradigm of habituation to electric shock that does not require animal manipulation such as tethering or decapitation. Moreover, long-lasting memory can be induced with a spaced training procedure with properties similar to long-term habituation parameters defined in other systems (Beck and Rankin 1997; Rose and Rankin 2001). The simplicity of this paradigm makes it suitable to conduct genetic screens aiming to elucidate the molecular basis of short- and long-term habituation.

Data from all paradigms evaluated to date suggest that the default response to repeated unpaired stimulus presentation in Drosophila is habituation, even if the stimulus is as noxious as electric shock (this study) or ethanol vapor (Cho et al. 2004). In both of these cases and other habituation paradigms in Drosophila (Corfas and Dudai 1989; Engel and Wu 1996; Jin et al. 1998; Engel et al. 2000) and other models (Harris 1943; Thomson and Spencer 1966; Hawkins 1988; Rose and Rankin 2001; Deshmukh and Bhalla 2003; Siniatchkin et al. 2003), emergence of habituation was observed after a "refractory" period or number of stimuli, during which animals responded as if they were naive. This suggests that within this time frame, processes that normally prevent premature habituation become inactivated, ones that mediate habituation are activated, or both. The refractory period may reflect the time necessary to evaluate a stimulus with respect to its saliency, novelty, and potential association with other stimuli prior to decreasing responsiveness toward it. Shortening or eliminating the refractory period would precipitate premature habituation. Our results strongly indicate that the MBs, especially the $\alpha / \beta$ lobes, play an important role in inhibition of premature habituation to electric shock stimuli. Therefore, we propose that processes within the $\alpha / \beta$ lobes protect from premature habituation, in essence ascertaining an adequate refractory period for stimulus evaluation.

The inability to dishabituate animals with abrogated $\mathrm{MB}$ function is in agreement with the proposed function of these neurons in stimulus evaluation and response modification. Olfactory and shock stimuli have been proposed to converge in the MBs (Roman and Davis 2001; Heisenberg 2003), a condition necessary for associative olfactory learning. Then, although the dishabituating olfactory stimulus reaches the MBs, the alteration in response it evokes cannot be transmitted to follower neurons if the $\alpha / \beta$ lobes are synaptically silenced or structurally impaired. Therefore, the attenuated response is not altered because the MBs are the blocked component of a neuronal circuit essential for manifestation of the response.

Interestingly, it is the $\alpha / \beta$ lobes again that are involved in habituation to ethanol vapor-induced startle (Cho et al. 2004). However, silencing $\alpha / \beta$-lobe synapses precipitated premature habituation to electric shock, but impaired or delayed habituation to ethanol vapor. These opposing effects could arise because two different drivers with incompletely overlapping expression patterns in $\alpha / \beta$ neurons (11Y [this study] and $17 \mathrm{~d}$ [Cho et al. 2004]) were used. Alternatively, whether the $\alpha / \beta$ lobes prevent or promote habituation to repetitive stimulation may depend on the properties of the stimulus itself. Structural and functional perturbation of the MBs precipitated premature habituation of olfactory responses in a simple aversion or attraction task (Acevedo 2004), suggesting that it is not the shock versus olfactory stimuli that underlie the opposing responses. Rather, it is likely that properties of the stimulus, such as intensity, salience, and context, that encompass the intrinsic differences of each experimental paradigm may account for the difference. Consistent with this notion, mutations in the adenylyl cyclase-encoding gene rutabaga have been reported to yield opposite effects in particular habituation paradigms in Drosophila (Duerr and Quinn 1982; Rees and Spatz 1989; Engel and Wu 1996). This likely reflects multiple and potentially paradigm-specific molecular mechanisms underlying habituation. Nevertheless, it is highly significant that the $\alpha / \beta$ lobes of the MBs were implicated in habituation to very different stimuli in distinct experimental paradigms, illustrating the importance of these neurons in evaluation of recurrent stimulation and mediation of the appropriate response.

The $\alpha / \beta$ lobes of the MBs are not likely to be the only locus 
in the brain required for protection from premature habituation. Since blocking neurotransmission from these neurons results in a phenotype similar to complete $\mathrm{MB}$ ablation, neurons in other brain areas receiving signals from $\alpha / \beta$ lobes are also involved in delaying, preventing, or modifying the default habituation response to repetitive stimulation. Such neurons may be involved in the motor aspect of attenuating the response to the stimulus. We propose a model in which the $\mathrm{MB} \alpha / \beta$ lobes function as evaluation centers monitoring stimuli, their properties, relationships among them, and the context within which they are encountered. Such information is communicated to other brain areas perhaps involved in direct responses to stimuli, where such responses are modified in an experience-dependent manner. One such brain locus may be the Lateral Horn, which is known to mediate direct responses to odors and other stimuli (Tanaka et al. 2004) and is reciprocally connected with the MBs (Ito et al. 1998). This model is consistent with other properties of the MBs such as their involvement in context generalization, which as for protection from habituation, also involves deriving and maintaining relationships between stimuli and requires anticipating future events based on a past experience (Heisenberg 2003). Moreover, the proposed inhibitory role of the MBs is consistent with their involvement in suppression of locomotor activity and generation, regulation, and coordination of motor programs (Martin et al. 1998; Besson and Martin 2005).

Conditioned aversion of an odor is mediated by pairing it to electric shock in the associative olfactory learning and memory paradigm (Tully and Quinn 1985). Given our results and the dependence of olfactory learning on the MBs (Roman and Davis 2001; Waddell and Quinn 2001; Heisenberg 2003), it is predicted that structurally or functionally impairing these neurons would result in premature habituation to the unconditioned stimulus (shock). This is likely to impair its temporal and contextual association with the odor, thus inhibiting learning. In agreement with this, animals with ablated MBs are profoundly unable to learn in this odor/shock association paradigm (deBelle and Heisenberg 1994). However, silencing MB synapses precipitated failure to learn in an appetitive associative learning paradigm pairing odors with sugar reward (Schwaerzel et al. 2003). These results suggest that attenuated shock avoidance is not the primary reason animals with perturbed MBs fail to learn, unless they also habituate prematurely to the odors or the sugar reward as well.

It is interesting that the $\alpha / \beta$ lobes have been associated with long-term memory (LTM) formation (Pascual and Preat 2001; Yu et al. 2006), and our results uncovered a novel function of these neurons in protection from premature habituation, a function likely to be requisite for learning. Although it is possible that the same neurons share these two functions, it is also likely that the distinct subsets of $\alpha / \beta$ neurons previously described (Strausfeld et al. 2003) contribute differentially to them. At the moment, it is unclear which $\alpha / \beta$ subsets are marked by the GAL4 drivers we used, but if the two functions are housed within the same neurons, it would be of interest to determine whether they use distinct or similar biochemistries. Our results predict that some gene(s) preferentially expressed in the $\alpha / \beta$ lobes may be specifically involved in protection from premature habituation to shock and perhaps not in LTM. The shock habituation assay we describe can provide a facile method in identification and characterization of such putative mutants.

\section{Materials and Methods}

\section{Drosophila strains}

Drosophila were cultured in standard wheat-flour-sugar food supplemented with soy flour and $\mathrm{CaCl}_{2}$ at $21^{\circ}-23^{\circ} \mathrm{C}$, unless specified otherwise. Animals able to express the tetanus toxin light chain transgene (UAS-TNT) (Sweeney et al. 1995) were raised at $18^{\circ} \mathrm{C}$ until hatching, then placed at $20^{\circ}-22^{\circ} \mathrm{C}$ for $2 \mathrm{~d}$ prior to testing. The control strain Berlin and the mushroom body miniature $\left(\mathrm{mbm}^{1}\right)$ mutants have been described previously (Heisenberg et al. 1985). Hydroxyurea (HU) ablated flies were prepared as described previously (Philip et al. 2001). Each batch of HUtreated adults was monitored histologically for the extent of mushroom body ablation along with $\mathrm{mbm}^{1}$ flies as described (Acevedo et al. 2007). Control flies carrying the $w^{1118}$ mutation had been backcrossed to the Canton-Special strain for at least 10 generations ( $w^{1118}$ strain). Transgenes used to block neurotransmission were UAS-TNT (Keller et al. 2002) and UAS-shits bearing a temperature-sensitive mutation of the dynamin encoded by the gene shibire (Kitamoto 2001). Tetanus toxin light chain prevents neurotransmitter release by cleaving synaptobrevin, a protein required for synaptic vesicle docking on presynaptic neurons (Humeau et al. 2000). In contrast, inactivation of the temperaturesensitive dynamin Shibire ${ }^{\text {ts }}$ is thought to prevent neurotransmitter recycling to the presynaptic neurons, causing their functional depletion (Kitamoto 2001). The Gal4 driver lines were P247, 201Y, c772, and H24 (Zars et al. 2000; Mershin et al. 2004), the reported $\gamma$-lobe-specific 1471 (Isabel et al. 2004) and NP1131 (Akalal et al. 2006), as well as the iACT driver GH146 (Stocker et al. 1997). The expression pattern and initial characterization of drivers $11 \mathrm{Y}$, c507, and $078 \mathrm{Y}$ can be found at http://www.flytrap.org, where these lines were obtained from J.D. Armstrong (University of Edinburgh, Scotland). All strains had been backcrossed to the Cantonised- $w^{1118}$ for four to six generations prior to use in our behavioral experiments.

\section{Behavioral analyses}

All flies used in behavioral experiments were tested 3-5 d after emergence. To obtain flies expressing tetanus light chain or the transgenic Shibire ${ }^{\mathrm{ts}}$, males of the Gal4 driver lines were crossed en masse to UAS-TNT and UAS-shits females or the parental $w^{1118}$, and the progeny was collected and tested 3-5 d after emergence. Control experiments demonstrated that progeny of the reverse crosses, where the female parents were from the Gal4 driver line, gave identical results with flies where the driver was derived paternally. All experiments were performed under dim red light at $25^{\circ} \mathrm{C}$ and $65 \%-70 \%$ relative humidity.

\section{Electroshock avoidance}

Experiments were performed under the conditions described above. Approximately 50 flies were placed at the choice point of a T-maze to choose for $90 \mathrm{sec}$ between an electrified and an otherwise identical inert standard copper grid. In the electrified grid, $45 \mathrm{~V}$ shocks were delivered every $4 \mathrm{sec}$, each lasting $1.2 \mathrm{sec}$ (unless indicated otherwise). The performance index (PI) was calculated as the fraction of flies avoiding the electrified grid minus the fraction of flies that do not.

\section{Habituation to electroshock}

Habituation to electric shock experiments were performed under the conditions described above. For the training phase $\sim 50$ flies were sequestered in the upper arm of a standard T-maze lined with an electrifiable grid. They were exposed to several 1.2-sec electric shocks at $45-90 \mathrm{~V}$ as indicated. Control experiments demonstrated that $45 \mathrm{~V}$ shocks are robustly avoided by wild-type strains and mushroom body ablated flies, but are mild enough to be easily habituated to. Multiple shocks were delivered with a 4-sec interstimulus interval. Air was not drawn through the tube during training to avoid association of the shocks with air. After a 30-sec rest and $\sim 120 \mathrm{sec}$ for transfer to the lower part of the maze, the flies were tested by choosing between an electrified and an inert grid. Therefore, the earliest measures of post-

\section{Learning \& Memory}


training responses are $2-3 \mathrm{~min}$ after the flies received the last training stimulus. Testing was done at the same voltage $(45,60$, or $90 \mathrm{~V}$ ) as for training. During the 90 -sec choice period, $17-18$ 1.2 -sec stimuli were delivered to the electrified arm of the maze. At the end of the choice period, the flies in each arm were trapped and counted, and a performance index was calculated as above. For long-term habituation, each training cycle consisted of 15 1.2-sec shocks at $45 \mathrm{~V}$. Animals were retained in the training tubes during the inter-training intervals (ITI). For memory tests 30-90 min post-training, trained animals were transferred to their holding vials before testing and were kept in the dark at $25^{\circ} \mathrm{C}$ and $65 \%-70 \%$ relative humidity.

Dishabituation was achieved by exposing the flies to benzaldehyde (500 $\mu \mathrm{L}$ of BNZ evaporating into an air stream passing over the liquid odorant at $500 \mathrm{~mL} / \mathrm{sec})$, or 3-octanol $(1000 \mu \mathrm{L}$ into an air stream passing over the liquid odorant at $500 \mathrm{~mL} / \mathrm{sec}$ ) for $10 \mathrm{sec}$ immediately after the "training phase." For spontaneous recovery, a resting period as indicated was allowed in the upper part of the maze between the training and testing phases. The performance index (PI) was calculated as described above.

\section{Statistical analysis}

Untransformed (raw) data were analyzed parametrically with the JMP3.1 statistical software package (SAS Institute Inc.).

\section{Acknowledgments}

We thank J. Grau and Sumana Datta for valuable discussions and suggestions, and Courtney Swayze and Maria Anezaki for stock maintenance and husbandry. This work was supported by $\mathrm{Na}-$ tional Science Foundation Grant IBN-0080687 and European Commission Marie Curie IRG-CT-003570.

\section{References}

Acevedo, S.F. 2004. "The dynamics, interactions and phenotypes associated with the three members of the 14-3-3 family in Drosophila melanogaster." Ph.D. thesis, Texas A\&M University, College Station, TX.

Acevedo, S.F., Froudarakis, E.I., Tsiorva, A.A., and Skoulakis, E.M.C. 2007. Distinct neuronal circuits mediate experience-dependent, non-associative osmotactic responses in Drosophila. Mol. Cell. Neurosci. 34: 378-389.

Adler, L.E., Freedman, R., Ross, R.G., Olincy, A., and Waldo, M. 1999. Elementary phenotypes in the neurobiological and genetic study of schizophrenia. Biol. Psychiatry 46: 8-18.

Akalal, D.B., Wilson, C.F., Zong, L., Tanaka, N.K., Ito, K., and Davis, R.L. 2006. Roles for Drosophila mushroom body neurons in olfactory learning and memory. Learn. Mem. 13: 659-668.

Asztalos, Z., vonWegener, J., Wustmann, G., Dombradi, V., Gausz, J., Spatz, H.-C., and Friedrich, P. 1993. Protein phosphatase 1-deficient mutant Drosophila is affected in habituation and associative learning. J. Neurosci. 13: 924-930.

Beck, C.D.O. and Rankin, C.H. 1997. Long term habituation is produced by distributed training at long ISIs and not by massed training or short ISIs in Caenorabdhitis elegans. Anim. Learn. Behav. 25: 446-457.

Besson, M. and Martin, J.R. 2005. Centrophobism/thigmotaxis, a new role for the mushroom bodies in Drosophila. J. Neurobiol. 62: $386-396$

Boynton, S. and Tully, T. 1992. latheo, a new gene involved in associative learning and memory in Drosophila melanogaster identified from $P$ element mutagenesis. Genetics 131: 655-672.

Burrell, B.D. and Sahley, C.L. 1998. Serotonin depletion does not prevent intrinsic sensitization in the leech. Learn. Mem. 6: 509-520.

Cho, W., Heberlein, U., and Wolf, F.W. 2004. Habituation of an odorant-induced startle response in Drosophila. Genes Brain Behav. 3: $127-137$.

Corfas, G. and Dudai, Y. 1989. Habituation and dishabituation of a cleaning reflex in normal and mutant Drosophila. J. Neurosci. 9: $56-62$.

Crittenden, J.R., Skoulakis, E.M.C., Han, K.-A., Kalderon, D., and Davis, R.L. 1998. Tripartite mushroom body architecture revealed by antigenic markers. Learn. Mem. 5: 38-51.

Davis, R.L. 2005. Olfactory memory formation in Drosophila: From molecular to systems neuroscience. Annu. Rev. Neurosci. 28: 275-302.
deBelle, S.J. and Heisenberg, M. 1994. Associative odor learning in Drosophila is abolished by chemical ablation of mushroom bodies. Science 263: 692-695.

Deshmukh, S.S. and Bhalla, U.S. 2003. Representation of odor habituation and timing in the hippocampus. J. Neurosci. 23: $1903-1915$.

Duerr, J.S. and Quinn, W.G. 1982. Three Drosophila mutations that block associative learning also affect habituation and sensitization. Proc. Natl. Acad. Sci. 79: 3646-3650.

Engel, J.E. and Wu, C.-F. 1996. Altered habituation of an identified escape circuit in Drosophila memory mutants. J. Neurosci. 16: $3486-3499$.

Engel, J.E., Xie, X.-J., Sokolowski, M.B., and Wu, C.-F. 2000. A CGMP-dependent protein kinase gene, foraging, modifies habituation-like response decrement of the Giant Fiber Escape Circuit in Drosophila. Learn. Mem. 7: 341-352.

Ezzeddine, Y. and Glanzman, D.L. 2003. Prolonged habituation of the gill-withdrawal reflex in Aplysia depends on protein synthesis, protein phosphatase activity, and postsynaptic glutamate receptors. J. Neurosci. 23: 9585-9594.

Freedman, R., Waldo, M., Bickford-Wimer, P., and Nagamoto, H. 1991. Elementary neuronal dysfunctions in schizophrenia. Schizophr. Res. 4: $233-243$.

Gillberg, C. 2003. Deficits in attention, motor control and perception: A brief review. Arch. Dis. Child. 88: 904-910.

Groves, P.M. and Thomson, R.F. 1970. Habituation: A dual-process theory. Psychol. Rev. 77: 419-450.

Harris, J.D. 1943. Habituatory response decrement in the intact organism. Psychol. Bull. 40: 385-422.

Hawkins, R.D. 1988. A simple circuit model for higher-order features of classical conditioning. In Neural models of plasticity (eds. J.H. Byrne and W.O. Berry), pp. 73-93. Academic Press, San Diego.

Heisenberg, M. 2003. Mushroom body memoir: From maps to models. Nat. Rev. Neurosci. 4: 266-275.

Heisenberg, M., Borst, A., Wagner, S., and Byers, D. 1985. Drosophila mushroom body mutants are deficient in olfactory learning. $J$. Neurogenet. 2: 1-30.

Humeau, Y., Doussau, F., Grant, N.J., and Poulain, B. 2000. How botulinum and tetanus neurotoxins block neurotransmitter release. Biochimie 82: 427-446.

Ilius, M., Wolf, R., and Heisenberg, M. 1994. The central complex of Drosophila melanogaster is involved in flight control: Studies on mutants and mosaics of the gene ellipsoid body open. J. Neurogenet. 9: $189-206$.

Isabel, G., Pascual, A., and Preat, T. 2004. Exclusive consolidated memory phases in Drosophila. Science 304: 1024-1027.

Ito, K., Suzuki, K., Estes, P., Ramaswami, M., Yamamoto, D., and Strausfeld, N.J. 1998. The organization of extrinsic neurons and their implications in the functional roles of the mushroom bodies of Drosophila melanogaster Meigen. Learn. Mem. 5: 52-77.

Jin, P., Griffith, L.C., and Murphey, R.K. 1998. Presynaptic calcium/calmodulin-dependent protein kinase II regulates habituation of a simple reflex in adult Drosophila. J. Neurosci. 18: $8955-8964$.

Keller, A., Sweeney, S.T., Zars, T., O'Kane, C.J., and Heisenberg, M. 2002. Targeted expression of tetanus neurotoxin interferes with behavioral responses to sensory input in Drosophila. J. Neurobiol. 50: 221-233.

Kitamoto, T. 2001. Conditional modification of behavior in Drosophila by targeted expression of a temperature-sensitive shibire allele in defined neurons. J. Neurobiol. 47: 81-92.

Mackintosh, N.J. 1974. Generalization. In The psychology of animal learning, pp. 485-542. Academic Press, New York.

Marcus, E.A., Nolen, T.G., Rankin, C.H., and Carew, T.J. 1988. Behavioral dissociation of dishabituation, sensitization and inhibition in Aplysia. Science 241: 210-213.

Martin, J.R., Ernst, R., and Heisenberg, M. 1998. Mushroom bodies suppress locomotor activity in Drosophila melanogaster. Learn. Mem. 5: $179-191$.

Martin, J.R., Raabe, T., and Heisenberg, M. 1999. Central complex substructures are required for the maintenance of locomotor activity in Drosophila melanogaster. J. Comp. Physiol. [A] 185: 277-288.

Martin, J.R., Faure, P., and Ernst, R. 2001. The power law distribution for walking-time intervals correlates with the ellipsoid-body in Drosophila. J. Neurogenet. 15: 205-219.

McGuire, S.E., Deshazer, M., and Davis, R.L. 2005. Thirty years of olfactory learning and memory research in Drosophila melanogaster. Prog. Neurobiol. 76: 328-374.

Meincke, U., Light, G.A., Geyer, M.A., Braff, D.L., and Gouzoulis-Mayfrank, E. 2004. Sensitization and habituation of the acoustic startle reflex in patients with schizophrenia. Psychiatry Res. 126: $51-61$.

Mershin, A., Pavlopoulos, E., Fitch, O., Braden, B.C., Nanopoulos, D.V., 
and Skoulakis, E.M.C. 2004. Learning and memory deficits upon TAU accumulation in Drosophila mushroom body neurons. Learn. Mem. 11: 277-287.

Michel, C.I., Kraft, R., and Restifo, L.L. 2004. Defective neuronal development in the mushroom bodies of Drosophila fragile X mental retardation 1 mutants. J. Neurosci. 24: 1175-1180.

Mihalek, R.M., Jones, C.J., and Tully, T. 1997. The Drosophila mutation turnip has pleiotropic behavioral effects and does not specifically affect learning. Learn. Mem. 3: 425-444.

Pascual, A. and Preat, T. 2001. Localization of long-term memory within the Drosophila mushroom body. Science 294: 1115-1117.

Philip, N., Acevedo, S., and Skoulakis, E.M.C. 2001. Conditional rescue of olfactory learning and memory defects in mutants of the 14-3-3̧ gene leonardo. J. Neurosci. 21: 8417-8425.

Rankin, C.H. 2000. Context conditioning in habituation in the nematode Caenorhabditis elegans. Behav. Neurosci. 114: 496-505.

Rankin, C.H. and Broster, B.S. 1992. Factors affecting habituation and recovery from habituation in the nematode Caenorhabditis elegans. Behav. Neurosci. 106: 239-249.

Rees, C.T. and Spatz, H.-C. 1989. Habituation of the landing response of Drosophila wild-type and mutants defective in olfactory learning. $J$. Neurogenet. 5: 105-118.

Roman, G. and Davis, R.L. 2001. Molecular biology and anatomy of Drosophila olfactory associative learning. Bioessays 23: 571-581.

Rose, J.K. and Rankin, C.H. 2001. Analyses of habituation in Caenorhabditis elegans. Learn. Mem. 8: 63-69.

Schwaerzel, M., Monastirioti, M., Scholz, H., Friggi-Grelin, F., Birman, S., and Heisenberg, M. 2003. Dopamine and octopamine differentiate between aversive and appetitive olfactory memories in Drosophila. J. Neurosci. 23: 10495-10502.

Siniatchkin, M., Kropp, P., and Gerber, W.-D. 2003. What kind of habituation is impaired in migraine patients? Cephalalgia 23: $511-518$.

Skoulakis, E.M.C. and Davis, R.L. 1996. Olfactory learning deficits in mutants for leonardo, a Drosophila gene encoding a 14-3-3 protein. Neuron 17: 931-944.

Skoulakis, E.M.C. and Grammenoudi, S. 2006. Dunces and da Vincis: The genetics of learning and memory in Drosophila. Cell. Mol. Life Sci. 63: 6067-6078.

Skoulakis, E.M.C., Kalderon, D., and Davis, R.L. 1993. Preferential expression in mushroom bodies of the catalytic subunit of protein kinase A and its role in learning and memory. Neuron 11: 197-208.

Slaats-Willemse, D., Swaab-Barneveld, H., de Sonneville, L., van der Meulen, E., and Buitelaar, J. 2003. Deficient response inhibition as a cognitive endophenotype of ADHD. J. Am. Acad. Child Adolesc.
Psychiatry 42: 1242-1248.

Stocker, R.F., Heimbeck, G., Gendre, N., and de Belle, J.S. 1997. Neuroblast ablation in Drosophila P[GAL4] lines reveals the origin of olfactory interneurons. J. Neurobiol. 32: 443-456.

Strausfeld, N.J., Sinakevitch, I., and Vilinsky, I. 2003. The mushroom bodies of Drosophila melanogaster: An immunocytological and Golgi study of Kenyon cell organization in the calyces and lobes. Microsc. Res. Tech. 62: 132-150.

Strauss, R., Hanesch, U., Kinkelin, M., Wolf, R., and Heisenberg, M. 1992. No-bridge of Drosophila melanogaster: Portrait of a structural brain mutant of the central complex. J. Neurogenet. 8: 125-155.

Sweeney, S.T.B., Keane, K., Niemann, J., and O'Kane, C.J. 1995. Targeted expression of tetanus toxin light chain in Drosophila specifically eliminates synaptic transmission and causes behavioral defects. Neuron 14: 341-351.

Tanaka, N.K., Awasaki, T., Shimada, T., and Ito, K. 2004. Integration of chemosensory pathways in the Drosophila second-order olfactory centers. Curr. Biol. 14: 449-457.

Thomson, R.F. and Spencer, W.A. 1966. Habituation: A model phenomenon for the study of neuronal substrates of behavior. Psychol. Rev. 73: 16-43.

Thum, A.S., Knapek, S., Rister, J., Dierichs-Schmitt, E., Heisenberg, M., and Tanimoto, H. 2006. Differential potencies of effector genes in adult Drosophila. J. Comp. Neurol. 498: 194-203.

Tully, T. and Quinn, W. 1985. Classical conditioning and retention in normal and mutant Drosophila melanogaster. J. Comp. Physiol. [A] 157: 263-277.

Waddell, S. and Quinn, W.G. 2001. Flies, genes and learning. Annu. Rev. Neurosci. 24: 1283-1309.

Yang, M.Y., Wang, Z., MacPherson, M., Dow, J.A., and Kaiser, K. 2000. A novel Drosophila alkaline phosphatase specific to the ellipsoid body of the adult brain and the lower Malpighian (renal) tubule. Genetics 154: 285-297.

Yu, D., Ponomarev, A., and Davis, R.L. 2004. Altered representation of the spatial code for odors after olfactory classical conditioning: Memory trace formation by synaptic recruitment. Neuron 42: 437-449.

Yu, D., Akalal, D.B., and Davis, R.L. 2006. Drosophila $\alpha / \beta$ mushroom body neurons form a branch-specific, long-term cellular memory trace after spaced olfactory conditioning. Neuron 52: 845-855.

Zars, T., Fischer, M., Schulz, R., and Heisenberg, M. 2000. Localization of a short-term memory in Drosophila. Science 288: 672-675.

Received November 14, 2006; accepted in revised form March 13, 2007. 


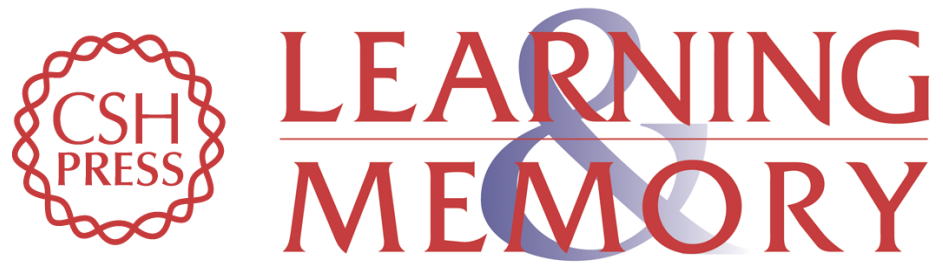

\section{Protection from premature habituation requires functional mushroom bodies in Drosophila}

Summer F. Acevedo, Emmanuil I. Froudarakis, Alexandros Kanellopoulos, et al.

Learn. Mem. 2007, 14:

Access the most recent version at doi:10.1101/lm.566007

References This article cites 66 articles, 25 of which can be accessed free at: http://learnmem.cshlp.org/content/14/5/376.full.html\#ref-list-1

License

Email Alerting Receive free email alerts when new articles cite this article - sign up in the box at the Service top right corner of the article or click here. 